\title{
Genetic Parameters for Sorghum Varieties in Different Population Densities
}

\author{
Kareema Mohamad Wuhaib, Banan Hassan Hadi, Wajeeha Abed Hassan \\ Department of Field Crops Sciences, College of Agriculture, University of Baghdad, Baghdad, Iraq \\ Email address: \\ Kareema522@yahoo.com (K. M. Wuhaib), bhd.1970@yahoo.com (B. H. Hadi), Wa.hassan69@yahoo.com (W. A. Hassan)
}

\section{To cite this article:}

Kareema Mohamad Wuhaib, Banan Hassan Hadi, Wajeeha Abed Hassan. Genetic Parameters for Sorghum Varieties in Different Population Densities. International Journal of Applied Agricultural Sciences. Vol. 3, No. 1, 2017, pp. 19-24. doi: 10.11648/j.ijaas.20170301.12

Received: August 26, 2016; Accepted: September 1, 2016; Published: February 13, 2017

\begin{abstract}
Experiments were carried out at Field Crop Dept., College of Agric., Univ. of Baghdad, during spring and fall seasons of 2013. The objectives were to estimate the:1- Phenotypic, genotypic and environmental variations, 2- Genotypic and phenotypic coefficient of variation and 3- broad sense heritability for seven traits days to 50\% anthesis (DTA), plant height $(\mathrm{PH})$, leaf area (LA), panicle length (PL), panicle weight (PW), crop growth rate (CGR), and grain yield per plant (GY). These traits and variances were recorded on three genotypes of sorghum (Sorghum bicolor L. Moench), Enqath, Rabih, and Kaffier. Results of statistical analysis showed that most traits had higher genotypic and phenotypic variances than environmental variance estimate, which indicated these traits were genotypic origin, and can be exploited in breeding programs. The genotypes exhibited varying degrees ratios of heritability for most traits. Such traits were responded positively to selection due to high broad sense heritability estimates. These data demonstrated high diversity for the traits studied of genotypes used.
\end{abstract}

Keywords: Heritability, GCV, PCV, Yield, Sorghum

\section{Introduction}

Sorghum (Sorghum bicolor L. Moench) is the second most important dry land crop for semi-arid tropics. It is grown in an area of 38 million hectares and with annual grain production of about 58 million tones and the productivity average reaches $1.5 \mathrm{ton} / \mathrm{ha}$. [1]. Although sorghum has an ability to tolerate many of environment stress, but it is affected by drought stress at the post flowering stage of growth $[2,3]$. Genetic variability for agronomic traits is a key component of breeding programs for broadening the gene pool of crops [4]. Heritability is measure of the phenotypic variance attributable to genetic causes and has predictive function in plant breeding. It provides information on the extent to which a particular genetic- morphological traits can be transmitted to successive generations, and influences the choice of selection procedures used by the plant breeder to decide which selection methods would be most useful to improve the trait, to predict gain from selection and to determine the relative importance of genetic effects $[5,6]$. Falconer and Mackay [7] reported that the most important function of heritability in genetic studies of quantitative traits is its predictive role to indicate the reliability of phenotypic value as guide to breeding value. Najeeb et al., [8] illustrated that traits with high heritability can easily be fixed with simple selection resulting in quick progress, but it has been emphasized that heritability alone has no practical importance without genetic advance. High genetic advance with high heritability estimates offers the most suitable condition for selection. High heritability of population is not enough to insure a final high yield/ plant without a high yield /plant originally in the base population or first cycle of selection of a scheme [9]. Tadesse et al., [10] indicated that the most traits had relatively higher genotypic and phenotypic variance components and little difference between PCV and indicating their variation has a genetic origin can be exploited for further breeding programs. Tesfamicheal et al., [11] found high magnitude of PCV and GCV for all agronomic traits studied. Individual and the combined analysis indicated that most traits had higher genotypic and phenotypic variance components than the environmental variance estimates, which is indicative that traits expression in this sorghum population was genetic and can be exploited in breeding programs [12]. Evaluation of components of variation and heritability for many traits will be facilitate improvement of crops, such as sorghum thus the 
objectives were to evaluate the variation in geneticmorphology and genetic- physiology traits in order to improve the yield of sorghum plants.

\section{Material and Methods}

\subsection{Plant Materials and Experiment Site}

The experiment materials consist of three genotypes: Enqath, Rabih and Kaffier. The sorghum genotypes were planted in two seasons Fall and Spring during march and July 2013 at the field of Field Crop Dept., College of Agric., Univ. of Baghdad in order to analyze some genetic parameters for yield and yield components of sorghum (Sorghum bicolor L. Moench). Population densities used were 60, 70, and 80 thousand plant/ha.

\subsubsection{Treatments and Experimental Design}

A split plot design was used, three main plots for genotypes with four replications. The sub plots were three population densities. The experiments were evaluated by using randomized complete block design (RCBD).

\subsubsection{Phenotypic Data}

At maturity the phenotypic data were measured on teen plants sampled at random from each plot; days to $50 \%$ anthesis (DTA), plant height (PH), leaves area(LA), Panicle length (PL), panicle weight (PW), crop growth rate (CGR) and yield per plant (YP).

\subsection{Statistical Analysis}

\subsubsection{Analysis of Variance}

To estimate the variation between genotypes and between plant populations the data recorded was analysed with ANOVA (Steel et al.) [13]. The least significant difference (LSD) were used to compare between means of treatments.

\subsubsection{Analysis of Genetic Variability and Estimation of Coefficients of Variation}

Genotypic and phenotypic variability were estimated as described by Singh and Chaudhary, [14] as follows

$$
\begin{aligned}
& \mathrm{PCV}=\left(\sqrt{\sigma^{2} P} / \bar{X}\right) \times 100 \\
& \mathrm{GCV}=\left(\sqrt{\sigma^{2} G} / \bar{X}\right) \times 100
\end{aligned}
$$

Components of variance $\left(\delta^{2} \mathrm{p}, \delta^{2} \mathrm{~g}, \delta^{2} \mathrm{e}\right)$ were estimated and used for the estimation of coefficients of variation (PCV, GCV):
$\delta^{2} \mathrm{p}$ : phenotypic variance
$\delta^{2}$ g: genotypic variance
$\delta^{2} \mathrm{e}$ : environment variance
$\bar{X}$ : grand mean
PCV: phenotypic coefficient of variation
GCV: genotypic coefficient of variation

\subsubsection{Estimation of Broad Sense Heritability}

To estimate broad sense heritability for the various traits the following formula was used.

$$
.^{2}{ }_{b . s}=\left(\delta^{2} g / \delta^{2} p\right) \times 100
$$

\section{Results and Discussion}

\subsection{Simple Variations}

Standard error and coefficients of variation for most traits are under the $20 \%$, thus the CV for all traits remained in an acceptable range (Tables $1,2, . .14$ ). The lower the values of $\mathrm{CV}$, the more the results are reliable because $\mathrm{CV}$ is an index of reliability and illustrate the magnitude of experimental error. Azize, [15] found that the CV was decrease as the plant populations increase for all traits he studied.

The performance of the genotypes varied in the two seasons indicated the diversity of the genotypes and their differences in environmental responses across two seasons per for all traits. This could be due to variability in the humidity, temperatures and other factors in the growing season. Similar findings were reported by $[16,17]$ in their studies.

Table 1. Mean of days to $50 \%$ anthesis for sorghum varieties in different population densities in spring season 2013.

\begin{tabular}{lllllll}
\hline \multicolumn{7}{l}{ Spring season } \\
\hline \multicolumn{7}{c}{ population densities } \\
varieties & 60000 & 70000 & 80000 & Means & C.V & SE \\
& $\mathrm{p} / \mathrm{h}$ & $\mathrm{p} / \mathrm{h}$ & $\mathrm{p} / \mathrm{h}$ & & & \\
Enqath & 59.00 & 63.75 & 67.00 & 63.25 & 5.61 & 1.02 \\
Rabih & 59.25 & 69.25 & 72.00 & 66.83 & 9.2 & 1.78 \\
Kafiar & 61.25 & 62.00 & 68.25 & 63.83 & 3.5 & 1.01 \\
L.S.D 0.05 & 2.79 & & & 1.44 & & \\
Means & 59.83 & 65.00 & 69.08 & & & \\
L.S.D & 2.25 & & & & & \\
C.V & 2.55 & 5.37 & 4.51 & & & \\
SE & 1.53 & 3.49 & 3.12 & & & \\
\hline
\end{tabular}

Table 2. Mean of days to $50 \%$ anthesis for sorghum varieties in different population densities in fall season 2013.

\begin{tabular}{lllllll}
\hline Fall season & \multicolumn{7}{c}{ population densities } \\
\hline \multirow{5}{*}{ varieties } & 60000 & 70000 & 80000 & Means & C.V & SE \\
& $\mathrm{p} / \mathrm{h}$ & $\mathrm{p} / \mathrm{h}$ & $\mathrm{p} / \mathrm{h}$ & & & \\
Enqath & 62.00 & 65.75 & 71.25 & 66.33 & 1.96 & 1.23 \\
Rabih & 63.00 & 73.50 & 77.25 & 71.25 & 6.58 & 4.45 \\
Kafiar & 63.00 & 64.00 & 65.75 & 64.25 & 7.95 & 5.83 \\
L.S.D 0.05 & 1.97 & & & 1.34 & & \\
Means & 62.67 & 67.75 & 71.42 & & & \\
L.S.D 0.05 & 1.16 & & & & & \\
C.V & 6.31 & 8.94 & 2.97 & & & \\
SE & 4.19 & 6.37 & 1.91 & & & \\
\hline
\end{tabular}

Table 3. Mean of plant height (cm) for sorghum varieties in different population densities in spring season 2013.

\begin{tabular}{lllllll}
\hline Spring season & \multicolumn{7}{l}{ population densities } & & & & \\
\hline \multirow{2}{*}{ varieties } & 60000 & 70000 & 80000 & Means & C.V & SE \\
& $\mathrm{p} / \mathrm{h}$ & $\mathrm{p} / \mathrm{h}$ & $\mathrm{p} / \mathrm{h}$ & & & \\
Enqath & 107.00 & 124.42 & 135.22 & 122.22 & 10.31 & 3.62 \\
Rabih & 93.14 & 102.21 & 107.47 & 100.94 & 6.99 & 3.91 \\
Kafiar & 127.5 & 152.88 & 156.22 & 145.54 & 9.33 & 3.63 \\
L.S.D 0.05 & 4.88 & & & 2.97 & & \\
Means & 109.21 & 126.51 & 132.97 & & & \\
\hline
\end{tabular}




\begin{tabular}{|c|c|c|c|}
\hline \multicolumn{4}{|c|}{ Spring season } \\
\hline L.S.D 0.05 & 3.09 & & \\
\hline C.V & 13.67 & 17.34 & 15.84 \\
\hline $\mathrm{SE}$ & 4.31 & 6.33 & 6.08 \\
\hline
\end{tabular}

Table 4. Mean of plant height (cm) for sorghum varieties in different population densities in fall season 2013.

\begin{tabular}{lllllll}
\hline fall season & \multicolumn{7}{c}{ population densities } & & & & \\
\hline \multirow{5}{*}{ varieties } & 60000 & 70000 & 80000 & Means & C.V & SE \\
& $\mathrm{p} / \mathrm{h}$ & $\mathrm{p} / \mathrm{h}$ & $\mathrm{p} / \mathrm{h}$ & & & \\
Enqath & 120.24 & 138.79 & 171.63 & 143.56 & 15.61 & 6.47 \\
Rabih & 121.92 & 123.12 & 198.12 & 147.72 & 18.26 & 10.77 \\
Kafiar & 128.58 & 145.62 & 185.42 & 153.21 & 16.33 & 7.22 \\
L.S.D 0.05 & 4.69 & & & 1.95 & & \\
Means & 123.58 & 135.58 & 185.06 & & & \\
L.S.D 0.05 & 4.24 & & & & & \\
C.V & 7.39 & 4.29 & 6.21 & & & \\
SE & 2.90 & 1.53 & 3.32 & & & \\
\hline
\end{tabular}

Table 5. Mean of leaf area (cm) for sorghum varieties in different population densities in spring season 2013.

\begin{tabular}{lllllll}
\hline Spring season & \multicolumn{7}{c}{ population densities } & & & & \\
\hline \multirow{5}{*}{ varieties } & 60000 & 70000 & 80000 & Means & C.V & SE \\
& $\mathrm{p} / \mathrm{h}$ & $\mathrm{p} / \mathrm{h}$ & $\mathrm{p} / \mathrm{h}$ & & & \\
Enqath & 372.1 & 314.9 & 305.6 & 330.9 & 9.67 & 9.24 \\
Rabih & 368.7 & 362.2 & 332.9 & 354.6 & 9.11 & 8.68 \\
Kafiar & 312.3 & 269.2 & 254.2 & 278.9 & 10.37 & 8.33 \\
L.S.D 0.05 & 23.98 & & & 13.97 & & \\
Means & 351.1 & 315.5 & 297.6 & & & \\
L.S.D 0.05 & 16.58 & & & & & \\
C.V & 9.01 & 13.23 & 12.72 & & & \\
SE & 9.18 & 12.05 & 10.93 & & & \\
\hline
\end{tabular}

Table 6. Mean of leaf area (cm) for sorghum varieties in different population densities in fall season 2013.

\begin{tabular}{|c|c|c|c|c|c|c|}
\hline \multicolumn{7}{|l|}{ fall season } \\
\hline \multirow[t]{3}{*}{ varieties } & \multicolumn{3}{|c|}{ population densities } & Means & C.V & SE \\
\hline & 60000 & 70000 & $80000 \mathrm{p} / \mathrm{h}$ & & & \\
\hline & $\mathrm{p} / \mathrm{h}$ & $\mathrm{p} / \mathrm{h}$ & & & & \\
\hline Enqath & 402.7 & 309.5 & 252.4 & 321.5 & 20.12 & 16.46 \\
\hline Rabih & 364.7 & 374.8 & 340.9 & 360.1 & 8.27 & 8.59 \\
\hline Kafiar & 382.4 & 385.1 & 351.8 & 373.1 & 8.66 & 9.34 \\
\hline L.S.D 0.05 & 50.00 & & & 32.66 & & \\
\hline Means & 382.4 & 356.5 & 315.0 & & & \\
\hline L.S.D 0.05 & 24.69 & & & & & \\
\hline C.V & 6.57 & 16.72 & 15.75 & & & \\
\hline SE & 7.27 & 16.24 & 14.32 & & & \\
\hline
\end{tabular}

The analysis of variances indicated that differences existed among the plant populations for most traits indicated that they are highly variable.

The significant effects of cultivar $\times$ plant population interaction were observed in most traits, also indicated that the environmental conditions in the three populations influenced the performance of the genotypes. There were similar response for all traits to populations, all traits decrease as the populations increase. Non significance observed for some traits showed that the genotypes are genetically similar with regards to these traits, so selection for these traits will therefor show no impact to genetic improvement.
Table 7. Mean of panicle length $(\mathrm{cm})$ for sorghum varieties in different population densities in spring season 2013.

\begin{tabular}{|c|c|c|c|c|c|c|}
\hline \multicolumn{7}{|c|}{ spring season } \\
\hline \multirow[b]{2}{*}{ varieties } & \multicolumn{6}{|c|}{ population densities } \\
\hline & 60000 & 70000 & 80000 & Means & C.V & SE \\
\hline & $\begin{array}{l}\mathrm{p} / \mathrm{In} \\
33.55\end{array}$ & 29.62 & $\begin{array}{l}\mathrm{p} / \mathrm{I} \\
28.65\end{array}$ & & & 098 \\
\hline $\begin{array}{l}\text { Enqath } \\
\text { Rabih }\end{array}$ & 28.79 & 25.15 & $\begin{array}{l}28.65 \\
24.47\end{array}$ & 30.61 & $\begin{array}{l}11.06 \\
815\end{array}$ & $\begin{array}{l}0.90 \\
0.62\end{array}$ \\
\hline Kafiar & 17.07 & 16.05 & 15.07 & 1606 & 7.132 & 0.34 \\
\hline L.S.D 0.05 & n.s & & & 1.74 & & \\
\hline Means & 26.47 & 23.61 & 22.82 & & & \\
\hline L.S.D 0.05 & 0.78 & & & & & \\
\hline C.V & 28.17 & 25.64 & 27.22 & & & \\
\hline SE & 2.15 & 1.74 & 1.79 & & & \\
\hline
\end{tabular}

Table 8. Mean of panicle length $(\mathrm{cm})$ for sorghum varieties in different population densities in fall season 2013.

\begin{tabular}{|c|c|c|c|c|c|c|}
\hline \multicolumn{7}{|l|}{ fall season } \\
\hline \multirow[b]{2}{*}{ varieties } & \multicolumn{3}{|c|}{ population densities } & \multirow[b]{2}{*}{ Means } & \multirow[b]{2}{*}{ C.V } & \multirow[b]{2}{*}{$\mathrm{SE}$} \\
\hline & $\begin{array}{l}60000 \\
\mathrm{p} / \mathrm{h}\end{array}$ & $\begin{array}{l}70000 \\
\mathrm{p} / \mathrm{h}\end{array}$ & $\begin{array}{l}80000 \\
\mathrm{p} / \mathrm{h}\end{array}$ & & & \\
\hline Enqath & 33.50 & 28.93 & 16.14 & 26.19 & 20.08 & 2.27 \\
\hline Rabih & 30.55 & 26.06 & 14.64 & 23.75 & 19.67 & 2.03 \\
\hline Kafiar & 32.19 & 22.39 & 15.14 & 23.24 & 21.12 & 2.15 \\
\hline L.S.D 0.05 & 2.71 & & & 0.78 & & \\
\hline Means & 32.08 & 25.79 & 15.31 & & & \\
\hline L.S.D 0.05 & 2.63 & & & & & \\
\hline C.V & 4.54 & 14.07 & 6.31 & & & \\
\hline SE & 0.42 & 1.05 & 0.28 & & & \\
\hline
\end{tabular}

Table 9. Mean of panicle weight for sorghum varieties in different population densities in spring season 2013.

\begin{tabular}{lllllll}
\hline \multicolumn{7}{l}{ spring season } \\
\hline varieties & \multicolumn{7}{l}{ population densities } & & Means & C.V & SE \\
& 60000 & 70000 & $80000 \mathrm{p} / \mathrm{h}$ & & & \\
& $\mathrm{p} / \mathrm{h}$ & $\mathrm{p} / \mathrm{h}$ & & & & \\
Enqath & 125.7 & 100.5 & 77.6 & 101.3 & 21.41 & 6.26 \\
Rabih & 89.6 & 78.7 & 74.6 & 81.0 & 10.51 & 2.46 \\
Kafiar & 99.2 & 69.1 & 62.6 & 77.0 & 23.71 & 5.27 \\
L.S.D 0.05 & 21.54 & & & 6.12 & & \\
Means & 104.8 & 82.8 & 71.6 & & & \\
L.S.D 0.05 & 3.34 & & & & & \\
C.V & 15.92 & 19.13 & 13.56 & & & \\
SE & 4.81 & 4.57 & 2.81 & & & \\
\hline
\end{tabular}

Table 10. Mean of panicle weight (gm) for sorghum varieties in different population densities in fall season 2013.

\begin{tabular}{lllllll}
\hline fall season & \multicolumn{7}{l}{ means } & C.V & SE \\
\hline varieties & \multicolumn{2}{l}{ population densities } \\
& 60000 & 70000 & 80000 & & & \\
& $\mathrm{p} / \mathrm{h}$ & $\mathrm{p} / \mathrm{h}$ & $\mathrm{p} / \mathrm{h}$ & & & \\
Enqath & 122.8 & 91.1 & 80.1 & 98.0 & 19.74 & 5.87 \\
Rabih & 98.8 & 84.7 & 60.3 & 81.3 & 17.16 & 4.97 \\
Kafiar & 89.3 & 81.8 & 43.5 & 71.6 & 19.86 & 6.17 \\
L.S.D 0.05 & 9.43 & & & 5.66 & & \\
Means & 103.6 & 85.9 & 61.3 & & & \\
L.S.D 0.05 & 6.17 & & & & & \\
C.V & 15.81 & 7.87 & 20.00 & & & \\
SE & 4.7 & 1.97 & 4.60 & & & \\
\hline
\end{tabular}


Table 11. Mean of crop growth rate (gm/plant/day) for sorghum varieties in different population densities in spring season 2013.

\begin{tabular}{llllllll}
\hline spring season & \multicolumn{7}{l}{ population densities } \\
\hline \multirow{5}{*}{ varieties } & 60000 & 70000 & 80000 & means & C.V & SE \\
& $\mathrm{p} / \mathrm{h}$ & $\mathrm{p} / \mathrm{h}$ & $\mathrm{p} / \mathrm{h}$ & & & \\
Enqath & 2.11 & 1.73 & 1.20 & 1.68 & 24.02 & 0.12 \\
Rabih & 1.62 & 1.33 & 1.21 & 1.39 & 16.34 & 0.06 \\
Kafiar & 1.70 & 1.21 & 1.17 & 1.36 & 22.54 & 0.09 \\
L.S.D 0.05 & 0.24 & & & 0.13 & & \\
Means & 1.81 & 1.42 & 1.19 & & & \\
L.S.D 0.05 & 0.18 & & & & & \\
C.V & 13.98 & 20.4 & 10.63 & & & \\
SE & 0.07 & 0.08 & 0.04 & & & \\
\hline
\end{tabular}

Table 12. Mean of crop growth rate (gm/plant/day) for sorghum varieties in different population densities in fall season 2013.

\begin{tabular}{lllllll}
\hline fall season & \multicolumn{7}{l}{ Plant population } & & & & \\
\hline \multirow{2}{*}{ varieties } & 60000 & 70000 & 80000 & means & C.V & SE \\
& $\mathrm{p} / \mathrm{h}$ & $\mathrm{p} / \mathrm{h}$ & $\mathrm{p} / \mathrm{h}$ & & & \\
Enqath & 2.08 & 2.05 & 1.49 & 1.88 & 18.06 & 0.09 \\
Rabih & 1.38 & 2.02 & 1.18 & 1.53 & 18.41 & 0.08 \\
Kafiar & 1.25 & 1.79 & 1.02 & 1.36 & 17.97 & 0.06 \\
L.S.D 0.05 & 0.087 & & & 0.054 & & \\
Means & 1.57 & 1.95 & 1.23 & & & \\
L.S.D 0.05 & 0.08 & & & & & \\
C.V & 20.4 & 11.40 & 11.34 & & & \\
SE & 0.11 & 0.06 & 0.04 & & & \\
\hline
\end{tabular}

The cultivar Enqath give higher yield $(85.06 \mathrm{~g} / \mathrm{plant}$ and $89.65 \mathrm{~g}$ /plant) for two seasons respectively (tables 13,14$)$. It gives high yield due to increase in panicle weight $101.3 \mathrm{~g} / \mathrm{p}$, 98g/p(tables, 9, 10) CGR $1.68 \mathrm{~g} / \mathrm{d}, 1.88 \mathrm{~g} / \mathrm{d}$ (tables, 11, 12) panicle length $30.61 \mathrm{~cm}, 26.19 \mathrm{~cm}$ (tables, 7, 8) for two seasons respectively.

Table 13. Mean of grain yield (gm/plant) for sorghum varieties in different population densities in spring season 2013.

\begin{tabular}{lllllll}
\hline spring season & \multicolumn{7}{l}{ Plant population } & & & & \\
\hline \multirow{2}{*}{ varieties } & 60000 & 70000 & 80000 & Means & C.V & SE \\
& $\mathrm{p} / \mathrm{h}$ & $\mathrm{p} / \mathrm{h}$ & $\mathrm{p} / \mathrm{h}$ & & & \\
Enqath & 107.59 & 78.71 & 68.87 & 85.06 & 21.13 & 5.18 \\
Rabih & 84.60 & 63.93 & 59.37 & 69.30 & 17.35 & 3.47 \\
Kafiar & 80.33 & 70.00 & 50.84 & 67.06 & 20.58 & 3.99 \\
L.S.D 0.05 & 7.35 & & & 4.06 & & \\
Means & 90.84 & 70.88 & 59.70 & & & \\
L.S.D 0.05 & 5.49 & & & & & \\
C.V & 14.71 & 11.59 & 14.89 & & & \\
SE & 3.86 & 2.37 & 2.57 & & & \\
\hline
\end{tabular}

Table 14. Mean of grain yield (gm/plant) for sorghum varieties in different population densities in fall season 2013.

\begin{tabular}{llllllll}
\hline fall season & \multicolumn{7}{l}{ Plant population } \\
varieties & 60000 & 70000 & 80000 & means & C.V & SE \\
& $\mathrm{p} / \mathrm{h}$ & $\mathrm{p} / \mathrm{h}$ & $\mathrm{p} / \mathrm{h}$ & & & \\
Enqath & 107.18 & 85.03 & 76.73 & 89.65 & 15.88 & 4.11 \\
Rabih & 88.07 & 80.11 & 65.64 & 77.94 & 15.15 & 3.41 \\
Kafiar & 78.96 & 72.30 & 37.45 & 62.90 & 19.72 & 5.58 \\
L.S.D 0.05 & 7.47 & & & 4.31 & & \\
Means & 91.40 & 79.15 & 59.94 & & & \\
L.S.D 0.05 & 5.25 & & & & & \\
\hline
\end{tabular}

\begin{tabular}{llll}
\hline fall season & & & \\
\hline C.V & 14.35 & 30.33 & 9.49 \\
SE & 3.79 & 5.25 & 2.16 \\
\hline
\end{tabular}

The yield was decrease as the plant population increase due to competition among plants, that attributable to reduce in PW, CGR, PL, and LA. While the days to anthesis and plant height were increased as the populations increase (Tables, 1, 2, 3, 4).

A wide range of variation was recorded in days to anthesis between cultivars which range from 63.25 to 68.25 days, while populations it range from 59.83 to 69.08 days (table, 1, 2).

\subsection{Genotypic and Phenotypic Variation}

The results of estimated genetic variability for grain yield per plant and other traits are presented in table $(15,16.17$, $18,19,20)$. The genetic parameters were differences by. The CV for all traits was less than $20 \%$. The $\delta^{2} \mathrm{~g}$ was higher than $\delta^{2}$ e for most traits except LA in population $60000 \mathrm{p} / \mathrm{ha}$., and $70000 \mathrm{p} / \mathrm{ha}$., and PL in $80000 \mathrm{p} / \mathrm{ha}$., in fall season, (table 18 , $19,20)$ in spring only $50 \%$ days to anthesis and PL in $60000 \mathrm{p} / \mathrm{ha}$., and 80000p/ha., respectively (table 15, 16, 17). These results indicated that most traits were highly influenced by genetic. These results illustrated by the ratio of $\delta^{2} \mathrm{~g} / \delta^{2} \mathrm{e}$ which is highin most traits. Baktash and Wuhaib [18] found that the environmental variances were lower than genotypic, for three seasons in most traits in their studies.

Table 15. Genetic parameters for some traits of sorghum in spring season for $60000 \mathrm{p} / \mathrm{ha}$ population.

\begin{tabular}{|c|c|c|c|c|c|c|c|c|}
\hline traits & c.v & $\delta^{2} \mathbf{g}$ & $\delta^{2} \mathrm{e}$ & $\delta^{2} \mathbf{p}$ & $\delta^{2} g / \delta^{2} \mathrm{e}$ & P.C.V & G.C.V & $\mathbf{H}^{2}{ }_{\text {.b.s }}$ \\
\hline DTM & 2.07 & 1.14 & 1.53 & 2.66 & 0.75 & 2.73 & 1.76 & 0.43 \\
\hline $\mathrm{PH}$ & 2.29 & 297.29 & 6.29 & 303.59 & 47.26 & 15.95 & 15.79 & 0.97 \\
\hline LA & 3.75 & 988.91 & 174.31 & 1163.22 & 5.22 & 9.69 & 8.94 & 0.85 \\
\hline PL & 8.25 & 70.70 & 4.77 & 75.47 & 14.82 & 32.82 & 31.76 & 0.94 \\
\hline PW & 7.12 & 250.87 & 50.47 & 301.34 & 4.97 & 17.39 & 15.87 & 0.83 \\
\hline CGR & 7.82 & 0.065 & 0.02 & 0.085 & 3.25 & 16.14 & 14.12 & 0.77 \\
\hline GY & 5.67 & 208.34 & 26.53 & 234.87 & 7.85 & 16.87 & 15.89 & 0.887 \\
\hline
\end{tabular}

Table 16. Genetic parameters for some traits of sorghum in spring season for $70000 \mathrm{p} / \mathrm{ha}$ population.

\begin{tabular}{|c|c|c|c|c|c|c|c|c|}
\hline traits & c.v & $\delta^{2} \mathrm{~g}$ & $\delta^{2} \mathrm{e}$ & $\delta^{2} \mathbf{p}$ & $\delta^{2} g / \delta^{2} \mathrm{e}$ & P.C.V & G.C.V & $\mathbf{H}_{\text {.b.s }}^{2}$ \\
\hline DTM & 2.62 & 9.58 & 6.92 & 16.50 & 1.38 & 6.24 & 3.67 & 0.58 \\
\hline PH & 3.09 & 641.33 & 15.36 & 656.69 & 41.75 & 20.26 & 20.01 & 0.97 \\
\hline LA & 4.18 & 2121.45 & 174.23 & 2295.69 & 12.18 & 15.18 & 14.60 & 0.92 \\
\hline PL & 7.14 & 47.15 & 2.84 & 49.99 & 16.60 & 29.94 & 29.03 & 0.94 \\
\hline PW & 9.57 & 168.55 & 66.10 & 234.65 & 2.55 & 18.04 & 15.28 & 0.72 \\
\hline CGR & 10.69 & 0.065 & 0.023 & 0.088 & 2.83 & 20.89 & 17.94 & 0.74 \\
\hline GY & 8.10 & 46.96 & 32.98 & 79.95 & 1.42 & 12.62 & 9.67 & 0.587 \\
\hline
\end{tabular}

Table 17. Genetic parameters for some traits of sorghum in spring season for 80000 p/ha population.

\begin{tabular}{lllllllll}
\hline traits & $\mathbf{c . v}$ & \multicolumn{1}{c}{$\boldsymbol{\delta}^{\mathbf{2}} \mathbf{g}$} & \multicolumn{1}{c}{$\boldsymbol{\delta}^{\mathbf{2}} \mathbf{e}$} & \multicolumn{1}{c}{$\boldsymbol{\delta}^{\mathbf{2}} \mathbf{p}$} & \multicolumn{1}{c}{$\boldsymbol{\delta}^{\mathbf{2}} \mathbf{g} / \boldsymbol{\delta}^{\mathbf{2}} \mathbf{e}$} & P.C.V & G.C.V & $\mathbf{H}^{\mathbf{2}}$.b.s \\
\hline DTM & 2.88 & 5.77 & 3.97 & 9.75 & 1.45 & 4.52 & 3.47 & 0.59 \\
PH & 2.89 & 598.27 & 14.76 & 613.03 & 40.53 & 18.63 & 18.40 & 0.97 \\
LA & 6.82 & 1492.14 & 412.22 & 1904.36 & 3.62 & 14.66 & 12.98 & 0.78 \\
PL & 9.49 & 47.69 & 4.69 & 52.38 & 10.17 & 31.72 & 30.26 & 0.91 \\
PW & 9.23 & 52.18 & 43.72 & 95.89 & 1.19 & 13.67 & 10.08 & 0.54 \\
CGR & 3.27 & 0.006 & 0.002 & 0.008 & 4.00 & 3.33 & 0.656 & 0.75 \\
GY & 4.68 & 79.39 & 7.81 & 87.20 & 10.17 & 15.64 & 14.93 & 0.91 \\
\hline
\end{tabular}


Similar results were obtained for PCV and GCV. The GCV closed with the values of PCV indicated the most traits were studies were controlled genetically except the traits reported above. The little differences between PCV and GCV for traits indicated that the variation has a genetic origin (Sami et al.)[19]. High estimates of GCV and PCV were observed for CGR in fall (60000p/ha.), and LA (70000p/ha.). yield and panicle length $(80000 \mathrm{p} / \mathrm{ha}$.). In spring season, panicle length recorded higher values in all populations. We noticed that the variation in the traits contributed markedly to the total variability. Similar results were obtained by Hemlata Sharma et al. [20]. Low value of GCV and PCV were observed for PL (60000p/ha.), PH(70000p/ha.), and DTA (80000p/ha.), at fall season. In spring (table $15,16,17)$, the trait DTA in all populations was low. Similar results obtained by Godbharle et al. [21].

Table 18. Genetic parameters for some traits of sorghum in fall season for $60000 \mathrm{p} / \mathrm{ha}$ population.

\begin{tabular}{lllllllll}
\hline traits & $\mathbf{c . v}$ & \multicolumn{1}{c}{$\boldsymbol{\delta}^{\mathbf{2}} \mathbf{g}$} & \multicolumn{1}{c}{$\boldsymbol{\delta}^{\mathbf{2}} \mathbf{e}$} & \multicolumn{1}{c}{$\boldsymbol{\delta}^{\mathbf{2}} \mathbf{p}$} & \multicolumn{1}{c}{$\boldsymbol{\delta}^{\mathbf{2}} \mathbf{g} \boldsymbol{\delta}^{\mathbf{2}} \mathbf{e}$} & $\mathbf{P . C . V}$ & $\mathbf{G . C . V}$ & $\mathbf{H}_{\text {.b.s }}{ }^{2}$ \\
\hline DTM & 2.57 & 20.91 & 2.93 & 23.83 & 7.14 & 7.36 & 6.89 & 0.88 \\
PH & 1.66 & 131.82 & 5.10 & 136.92 & 25.84 & 8.61 & 8.45 & 0.96 \\
LA & 6.56 & 203.89 & 632.43 & 836.33 & 0.32 & 7.55 & 3.73 & 0.24 \\
PL & 1.71 & 2.11 & 0.300 & 2.416 & 7.05 & 4.84 & 4.53 & 0.88 \\
PW & 8.42 & 277.98 & 76.22 & 354.21 & 3.65 & 18.16 & 16.09 & 0.78 \\
CGR & 3.58 & 0.197 & 0.003 & 0.201 & 65.66 & 28.55 & 28.33 & 0.98 \\
GY & 5.08 & 202.06 & 21.59 & 223.65 & 9.36 & 16.36 & 15.55 & 0.90 \\
\hline
\end{tabular}

Table 19. Genetic parameters for some traits of sorghum in fall season for $70000 \mathrm{p} / \mathrm{ha}$ population.

\begin{tabular}{|c|c|c|c|c|c|c|c|c|}
\hline traits & c.v & $\delta^{2} \mathrm{~g}$ & $\delta^{2} \mathrm{e}$ & $\delta^{2} p$ & $\delta^{2} \mathrm{~g} / \delta^{2} \mathrm{e}$ & P.C.V & G.C.V & $\mathbf{H}^{2}{ }_{\text {.b.s }}$ \\
\hline DTM & 1.57 & 54.25 & 1.25 & 55.50 & 0.43 & & 10.34 & 0.97 \\
\hline $\mathrm{PH}$ & 1.44 & 18.67 & 3.15 & 21.83 & 5.93 & 3.781 & 3.49 & 0.86 \\
\hline LA & 16.47 & 819.16 & 3448.70 & 4267.94 & 0.24 & 18.33 & 8.03 & 0.19 \\
\hline PL & 4.91 & & 1.61 & & 42 & & & 0.87 \\
\hline PW & 7.21 & 12.86 & 38.38 & 51.24 & 0.34 & 8.34 & 4.18 & 0.25 \\
\hline CGR & 3.94 & 0.019 & 0.006 & 0.025 & 3.17 & 8.16 & 7.15 & 0.77 \\
\hline GY & 7.23 & 33.01 & 32.74 & 65.75 & 1.01 & 10.25 & 7.26 & 0.50 \\
\hline
\end{tabular}

Table 20. Genetic parameters for some traits of sorghum in fall season for $80000 \mathrm{p} / \mathrm{ha}$ population.

\begin{tabular}{lllrlllll}
\hline traits & $\mathbf{c . v}$ & \multicolumn{1}{c}{$\boldsymbol{\delta}^{\mathbf{2}} \mathbf{g}$} & \multicolumn{1}{c}{$\boldsymbol{\delta}^{\mathbf{2}} \mathbf{e}$} & \multicolumn{1}{c}{$\boldsymbol{\delta}^{\mathbf{2}} \mathbf{p}$} & \multicolumn{1}{c}{$\boldsymbol{\delta}^{\mathbf{2}} \mathbf{g} / \boldsymbol{\delta}^{\mathbf{2}} \mathbf{e}$} & P.C.V & $\mathbf{G . C . V}$ & $\mathbf{H}_{\text {.b.s }}^{\mathbf{2}}$ \\
\hline DTM & 1.77 & 1.61 & 1.31 & 2.92 & 1.23 & 2.66 & 1.97 & 0.55 \\
PH & 1.46 & 173.73 & 7.29 & 181.02 & 23.84 & 7.27 & 7.12 & 0.96 \\
LA & 5.19 & 2904.37 & 267.78 & 3172.16 & 10.85 & 17.88 & 17.11 & 0.92 \\
PL & 4.96 & 0.433 & 0.5815 & 1.02 & 0.74 & 6.58 & 4.29 & 0.43 \\
PW & 6.49 & 331.59 & 15.833 & 347.42 & 20.94 & 30.39 & 29.69 & 0.95 \\
CGR & 4.33 & 0.057 & 0.0029 & 0.059 & 19.66 & 19.89 & 19.37 & 0.95 \\
GY & 7.72 & 404.72 & 21.39 & 426.11 & 18.92 & 34.44 & 33.56 & 0.91 \\
\hline
\end{tabular}

\subsection{Heritability}

The results of broad sense heritability for yield and other traits are presented in tables $(15,16,17,18,19,20)$. The most of traits had high heritability above $80 \%$, another traits had medium heritability, where as LA had low heritability in fall season (tables 18, 19, 20), but it had high in spring (tables 15, 16, 17) due to the differences in environment which influence the plant growth. Also the DTA had high heritability in spring than fall due to differences in environment between fall and spring and between various populations. The high level of heritability indicates the preponderance of genetic variation which is less affected by environment. This result is in agreement with finding of Tomar et al. [22]. The phenotypic selection will be effective for these traits. The medium and low heritability mean that variations are attributed to high level of environmental effects and non- additive gene action was found and there were limited role for selection (Prabhakar [23]. Al-khazragy [24] pointed that the heritability is the importance of genetic parameters for selection trait in breeding program, as affecting in amount of genetic advance resulting from selection, the high heritability for trait which related with yield does not guarantee the required genetic gain unless this correlation be positive and high significant.

\section{Conclusion}

The success of breeding programs depends upon the genetic variation in the materials analyze. When genetic variability was greater, we can get higher heritability, so the chances of success to be achieved through selection were better. The results showed that sufficient genetic variability among genotypes was found. The amount of genetic variance were greater than environments, and contribute high level of phenotypic variance indicated that more of traits were genetically controlled. Greater genetic variance allowed improvement the traits. More of traits had high value of GCV and PCV. these data indicated that the variability could be exploited for successful identification of favorable genotypes for the traits desirable. There were high estimates of heritability for most traits, indicated that the superior genotype which possess better performance and high level of heritability for different traits.

\section{References}

[1] Food and Agriculture Organization Crop Production (FAOSTAT). 2014. World Sorghum Production and Utilization. FAO, Rome.

[2] Kebede, H., P. K. Subudhi, D. T., Rosenow, and H. T. Nguyen. 2001. Qauntittive traits loci influences drought tolerance grain sorghum (Sorghum bicolor L. Moench). Theoretical and applied genetics 103: 266-276.

[3] Tuinstra, M. R., E. M. Grote, P. B. Goldsbrough, and G. Ejeta. 1997. Genetic analysis of post-flowering drought tolerance and components of grain development in (Sorghum bicolor L. Moench). 3:439-448.

[4] Ahmad, S. Q., S. Khan, M. Khaffar, and F. Ahmad.2011. Genetic diversity analysis for yield and other parameters in maize (ZEA mays L.) genotypes. Asian journal of Agriculture Sciences. 3: 385-388.

[5] Tariq, A. S., Z. Akram, G. Shabbir, M. Gulfraz, K. S. Khan, M. S. Iqbal, and T. Mahmood.2012. Character association and inheritance studies of different sorghum genotypes for fodder yield and quality under irrigated and rain fed conditions. 
[6] Laghari, K. A., M. A. Afzal, A. A. Mirbahar. A. J. Pirzada, M. U. Dahot, and S. M. Mangrio.2010. Heritability studies of yield and yield associated traits in bread wheat. Pakistan Journal of Botany. 42: 111-115.

[7] Falconer, D. S. and T. F. C. Mackay. 1996. Introduction to quantitative genetics. $4^{\text {th }}$ ed. Benjamin Cummings, England, PP. 245-247.

[8] Najeeb, S., A. G. Rather, F. A. sheikh, and S. M. Razvi. 2009. Genetic variability, genotypic correlation and path coefficient analysis in maize under high altitude temperate ecology of Kashmir. Maize Genetic Cooperation Newsletter. 83: 1-8.

[9] Elsahookie, M. M. and K. M. Wuhaib.1990. Heritability and homeostasis of maize populations derived by different selection schemes. Mesopotamia J. of Agric. 22 (4): 7-16.

[10] Yohannes, T., M. Weldetsion, N. Abraha, E. Manyasa, and T. A braha.2015. Combine selection for earliness and yield in pedigree developed sorghum (Sorghum bicolor L. Moench) progenies in Eriterea. Plant Breed. Plant Breed. Genet. 3 (1): 18.

[11] Tesfamichael, A. S. M. Githiri, R. Kasili, A. Woldeamalak and A. B. Nyende.2015. genetic variation among sorghum (Sorghum bicolor L. Moench). landraces from Eritrea under post-flowering drought stress conditions. American Journal of plant sciences. 6: 1410-1424.

[12] Bello, D. A. M. Kadams, S. Y. Simon, and D. S. Mashi. 2007. Studies on genetic variability in cultivated sorghum(Sorghum bicolor L.) cultivars of adamava state Nigeria. Amer-Eruasian J. Agric. Sci. 2:297-302.

[13] Steel, R. G. D., J. H. Torrie, and D. A. Dickey.1997. Principles and Procedure of Statistics: A Biological Approach. $2^{\text {nd }}$ Edition. Mcgraw Hill Book Inc. New York. USA.

[14] Singh, P. K., and S. D. Chaudhary.1985. Biometrical methods in quantitative genetics analysis. Khalyni New Delhi, India,
PP. 318.

[15] Aziz F. O. J.2008. Breeding Sunflower, Sorghum and Maize by Honycomb. Ph. D. Dissertation, Dept. of Crop Sci., Coll. of Agric. Univ. of Baghdad.

[16] Sami, R. A., M. Y. Yeye. M. F. Ishiyaku, and I. S. Usman.2013. Heritability studies in some sweet sorghum (Sorghum bicolor L. moench) genotypes. J. Bio Agric. Healthcare.3 (17): 49-51.

[17] Baktash, F. Y., and K. M. Wuhaib.2003. Genotypic and phenotypic variances and correlations in several maize characters. The Iraqi J. of Agric. Sci. 34 (2): 91-100.

[18] Sharma, H., D. K. Jain, and V. Sharma.2006. Variability and path coefficient analysis in sorghum. Indian J. Agric. Res. 40 (4): $310-312$.

[19] Godbharle, A. R., A. W. More, and S. S. Ambekar. 2010. genetic variability and correlation studies in elite $-\mathrm{B}$ - and $-\mathrm{R}$ lines in kharif sorghum. Electronic J. of Plant Breeding. 1 (4): 989-993.

[20] Tomar, S. S., S. Sivakumar, and K. Ganesamurthy. 2012. Genetic variability and heritability studies for different quantitative traits in sweet sorghum (Sorghum bicolor L. Moench) genotypes. Electronic J. of Plant Breeding. 3(2): 806-810.

[21] Prabhakar.2003. genetic variability and correlation studies in F2 population of Rabi sorghum. J. Maha. Agric. Univ. 28 (2): 202-203.

[22] Al-Khazragy, B. H. 2006. Genetic Gain by Selection Dependence on Some Criteria of Selection Under Different of Nitrogen Fertilizer to Maize. MSc. Thesis, Dept. of Crop Sci., Coll. of Agric., Univ. of Baghdad. 